\title{
Grey Relational Analysis and Its Application Based on the Angle Perspective in Time Series
}

\author{
Guodong Xu, ${ }^{1}$ Peng Guo, ${ }^{1}$ Xuemei Li, ${ }^{2}$ and Yingying Jia ${ }^{1}$ \\ ${ }^{1}$ School of Management, Northwestern Polytechnical University, Xian 710129, China \\ ${ }^{2}$ College of Economics and Management, Nanjing University of Aeronautics and Astronautics, Nanjing 210016, China \\ Correspondence should be addressed to Guodong Xu; npuxgd@gmail.com
}

Received 20 May 2014; Revised 21 August 2014; Accepted 22 August 2014; Published 3 September 2014

Academic Editor: Li Weili

Copyright (C) 2014 Guodong Xu et al. This is an open access article distributed under the Creative Commons Attribution License, which permits unrestricted use, distribution, and reproduction in any medium, provided the original work is properly cited.

\begin{abstract}
The research scope of grey relational analysis has not been developed in recent years, and the exiting GRA models are all based on three perspectives, including distance perspective, slope perspective, and area perspective. Under this situation, a novel model called grey relational analysis based on angle perspective (GRAAP) was developed in this paper for the first time, and this model can expand the research scope of GRA. Like other GRA models, GRAAP not only has the properties of symmetry, uniqueness, comparability, and so forth but also can be used to make predictions, assessments, classifications, and so on. Finally, this novel model was proved to be rational and effective through a comparative study on the similar degree of four time series.
\end{abstract}

\section{Introduction}

In previous studies, some traditional methods, used to deal with the decision problems, usually need to rely on big sample data, such as linear programming [1] and regression analysis [2], but in the real-world there are also some other special cases with the characteristics of inefficient samples, instability, and irregularity, these special cases have taken so many difficulties for the decision-making. Under this situation, grey system theory (GST), aiming to solve this type of decision problems, has been developed; this theory can greatly utilize the partly known information in these cases to provide the support for the decision-makers [3]. And one important branch of GST, called grey relational analysis (GRA) [4], has been viewed as the most fundamental methodology of GST in decision-making, prediction, and so on $[5,6]$. Similar to GST, the research object of GRA can be the time series with inefficient sample and poor information, and its basic principle is to utilize the similarity of geometrical figure generated by different time series to uncover their relationship [7]. Specifically, the discrete values of time series in two-dimension coordinate system can be connected into the continuous broken line segments based on the thought of piecewise linear interpolation (PLI), and then their grey relational coefficient and relational degree can be calculated using some methods from traditional GRA; then, we can know about the relationships between these time series. In practice, GRA can be employed to generate, excavate, and abstract valuable information from the partially known information [8], that is to say, the adverse influences of poor date or short cycle systems can be greatly reduced to a certain extent through GRA. At present, this theory has been widely applied in many disciplines, such as economics, sociology, industry, agriculture, mining industry, traffic, education, medical science, ecology, water conservancy, geology, and aeronautics and astronautics $[9,10]$.

In view of the extensive applications, plenty of GRA models have been developed based on the grey relational axiom proposed by Professor Deng. These models can be divided into three categories according to their modeling basis. The first category, the most common category, was based on the distance between adjacent discrete values of the time series, the representative models, for example, Deng's grey relational model [11], T's grey relational model [12], the improved model of T's grey relational [5], the grey absolute degree of grey incidence model [13], the grey relative degree of grey incidence model, B's grey relational degree model [14], C's grey relational degree model [15], and so forth. The second 
category was based on the slope of the straight line generated by the discrete values of time series, the representative models included the grey absolute degree of grey incidence model [16] and the degree of grey slop incidence [17]. The third category was based on the area surrounded by different time series, the representative studies, for example, Liu analyzed the relationship between the absolute value and the relative increment in two different time series and then presented the thought that the relational degree between different time series could be measured by the area surrounded by these time series [18] and another grey relational model established by Guimerà et al. and also based on this thought [19].

In general, so many GRA models have been introduced by researchers as described above, and these models have also played an important role in dealing with the practical problems. However, there are still some other problems existing in these models, for example, some models do not have the properties of uniqueness [20], symmetry [21], parallelism [22], and so forth, and the modeling basis of GRA has not been developed in recent years, which still focused on the above three perspectives. Under these backgrounds, a novel model, called grey relational analysis based on angle perspective (GRAAP), will be introduced in this paper, and this model can greatly improve the accuracy and validity of the research results by making full use of the poor information existing in time series. Additionally, it has the properties of normativity, uniqueness, parallelism, and so forth. Finally, the paper proves the effectiveness and rationality of the model through the comparative study of four time series. And this novel model can also expand the research scope of GRA and enrich GST.

\section{Principle}

The research foundation of GRAAP was mainly based on two traditional GRA by Sun and Dang $[5,12]$; and its basic principle is to use the cointegrated similarity of angles to reveal the relationship between different time series.

For instance, assume that there are two time series $X$ and $X^{\prime}$, and they all have three coordinate points in the twodimensional coordinate system, that is, the points $A, B$, and $C$ corresponding to the time series $X$ and the points $A^{\prime}, B^{\prime}$, $C^{\prime}$, or $D^{\prime}$ corresponding to the time series $X^{\prime}$. And two curves can be created by connecting three coordinate points in every time series (Figure 1), and then, to the time series $X$, the angle $\alpha$ will be generated between line $A B$ and line $B C$, and to the time series $X^{\prime}$, if the corresponding coordinate point of the last observation time point $t_{3}$ lies on the $\operatorname{dot} C^{\prime}$, the angle $\alpha^{\prime}+\beta^{\prime}$ will be generated between line $A^{\prime} B^{\prime}$ and line $B^{\prime} C^{\prime}$, since the line $A B$ is parallel to the line $A^{\prime} B^{\prime}$, and the line $B C$ is also parallel to the line $B^{\prime} C^{\prime}$, the conclusion will be $\alpha=\alpha^{\prime}+\beta^{\prime}$, and the similarity of these two time series is higher. However, to the time series $X^{\prime}$, if the corresponding coordinate point of the last observation time point $t_{3}$ moves to the $\operatorname{dot} D^{\prime}$, the angle $\alpha^{\prime}$ will be generated, at this time, since the line $A B$ is parallel to the line $A^{\prime} B^{\prime}$, but the line $B C$ is not parallel to the line $B^{\prime} D^{\prime}$, the conclusion will be $\alpha>\alpha^{\prime}$, and the similarity of these two time series is lower.

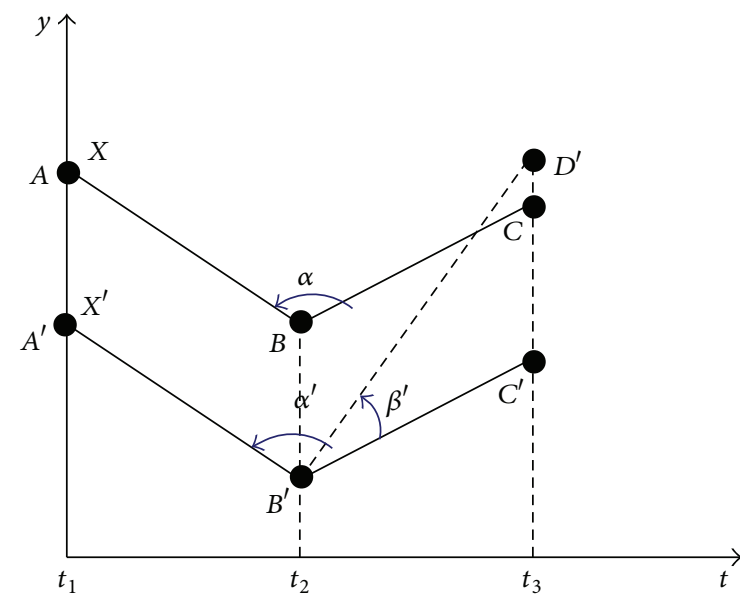

FIGURE 1: Schematic diagram of GRAAP.

In the above process, the relationship intensity between different time series mainly depends on the similarity of angle values determined by the positions of discrete coordinate of time series in two-dimension coordinate system. Based on this principle, a novel model called grey relational analysis based on the angle perspective (GRAAP) will be introduced, and there are two key challenges in the construction of this model, one is how to transform the traditional time series into the angle series, the other is how to calculate the grey relational coefficient between different series.

\section{Algorithm}

3.1. Details of the Algorithm of GRAAP. The details of the algorithm of GRAAP are presented as follows.

Step 1 (transforming the original time series). Time series is one kind of typical discrete sequences, and it can be expressed in many ways using some methods. In this paper, time series will be expressed as the form of piecewise function based on the thought of piecewise linear interpolation (PLI). PLI is an important thought which can help us to know about the characteristics and simplify the dimensions of the time series. For most time series, since they usually have the fixed observation time points, it can be viewed as an "automatic PLI," that is to say, this kind of series can determine the piecewise number by itself. Therefore, the time series can usually be expressed as the following formula:

$$
\begin{gathered}
X_{m}=\left\{\left(x_{m}\left(t_{1}\right), x_{m}\left(t_{2}\right)\right), \ldots,\left(x_{m}\left(t_{i-1}\right), x_{m}\left(t_{i}\right)\right), \ldots,\right. \\
\left.\left(x_{m}\left(t_{n-1}\right), x_{m}\left(t_{n}\right)\right)\right\},
\end{gathered}
$$

where $m$ denotes the number of time series; $n$ denotes the number of observing time points in one series; $t_{i}$ denotes the observation time points; $x_{m}\left(t_{i}\right)$ denotes the observation value of the $n$th time series at $t_{i}$; and $\left(x_{m}\left(t_{i-1}\right), x_{m}\left(t_{i}\right)\right)$ denotes the line segment with the starting value $x_{m}\left(t_{i-1}\right)$ and the final value $x_{m}\left(t_{i}\right)$ (Figure 2). 


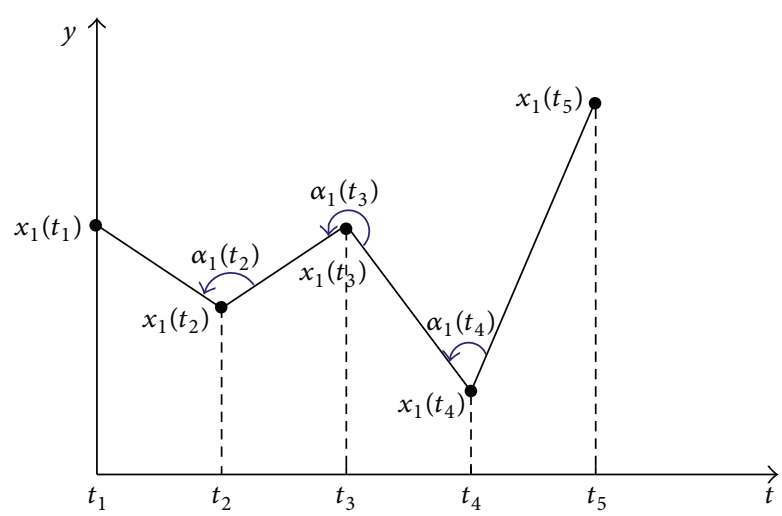

Figure 2: PLI of time series.

Definition 1. Set the time series that can be expressed as $A_{m}=\left\{\alpha_{m}\left(t_{2}\right), \alpha_{m}\left(t_{3}\right), \ldots, \alpha_{m}\left(t_{n-1}\right)\right\}$, one defines it as the corresponding angle series of the time series, and it can also be viewed as another normalization form of the time series, where $\alpha_{m}\left(t_{i}\right)$ denotes the counterclockwise angle between the line segments $\left(x_{m}\left(t_{i-1}\right), x_{m}\left(t_{i}\right)\right)$ and the $\left(x_{m}\left(t_{i}\right), x_{m}\left(t_{i+1}\right)\right)$ of the $m$ th time series.

In order to obtain the angle values in one angle series, set $\beta_{m}\left(t_{i}\right)$ that means the clockwise angle between the line segments $\left(x_{m}\left(t_{i-1}\right), x_{m}\left(t_{i}\right)\right)$ and $\left(x_{m}\left(t_{i}\right), x_{m}\left(t_{i+1}\right)\right)$ of the $m$ th time series; then the calculation method of $\alpha_{m}\left(t_{i}\right)$ will be presented as follows:

$$
\begin{aligned}
& \alpha_{m}\left(t_{k}\right) \\
& = \begin{cases}\arccos \frac{b^{2}+c^{2}-a^{2}}{2 b c} & 2 \leq k \leq n-1, \alpha_{m}\left(t_{k}\right) \leq \beta_{m}\left(t_{k}\right), \\
2 \pi-\arccos \frac{b^{2}+c^{2}-a^{2}}{2 b c} & 2 \leq k \leq n-1, \alpha_{m}\left(t_{k}\right)>\beta_{m}\left(t_{k}\right),\end{cases}
\end{aligned}
$$

where

$$
\begin{aligned}
& a=\sqrt{\left[x_{m}\left(t_{i+1}\right)-x_{m}\left(t_{i-1}\right)\right]^{2}+\left(t_{i+1}-t_{i-1}\right)^{2}}, \\
& b=\sqrt{\left[x_{m}\left(t_{i}\right)-x_{m}\left(t_{i-1}\right)\right]^{2}+\left(t_{i}-t_{i-1}\right)^{2}}, \\
& c=\sqrt{\left[x_{m}\left(t_{i+1}\right)-x_{m}\left(t_{i}\right)\right]^{2}+\left(t_{i+1}-t_{i}\right)^{2}} .
\end{aligned}
$$

Step 2 (calculating the angle increment). Assume there are only two time series on the interval $[p, q]$, their corresponding angle series are $A_{1}=\left\{\alpha_{1}\left(t_{2}\right), \alpha_{1}\left(t_{3}\right), \ldots, \alpha_{1}\left(t_{n-1}\right)\right\}$ and $A_{2}=\left\{\alpha_{2}\left(t_{2}\right), \alpha_{2}\left(t_{3}\right), \ldots, \alpha_{2}\left(t_{n-1}\right)\right\}$, respectively; set the following formula as the increment function of the corresponding angle series from $t_{k-1}$ to $t_{k}$ :

$$
y_{m}\left(t_{k}\right)=\alpha_{m}\left(t_{k}\right)-\alpha_{m}\left(t_{k-1}\right) \quad(k=3,4, \ldots, n-1) .
$$

The purpose of building the increment function is to know about the change regularity of the angle values between the adjacent moments in one time series, and it is also advantageous to effectively obtain the grey relational degree in Steps 3 and 4.
Step 3 (calculating the grey relational coefficient). Assume there are only two time series on the interval $[p, q]$, their corresponding angle series are $A_{1}=\left\{\alpha_{1}\left(t_{2}\right), \alpha_{1}\left(t_{3}\right), \ldots\right.$, $\left.\alpha_{1}\left(t_{n-1}\right)\right\}$ and $A_{2}=\left\{\alpha_{2}\left(t_{2}\right), \alpha_{2}\left(t_{3}\right), \ldots, \alpha_{2}\left(t_{n-1}\right)\right\}$, respectively; the following formula will be defined to calculate the relational coefficient between these two time series:

$$
\begin{aligned}
\xi\left(t_{k}\right)=(1 & +\frac{\left|y_{1}\left(t_{k}\right)-y_{2}\left(t_{k}\right)\right|}{2 \pi} \\
& \left.+\frac{1}{2}\left[1-\frac{\min \left(\left|y_{1}\left(t_{k}\right)\right|,\left|y_{2}\left(t_{k}\right)\right|\right)}{\max \left(\left|y_{1}\left(t_{k}\right)\right|,\left|y_{2}\left(t_{k}\right)\right|\right)}\right]\right)^{-1} .
\end{aligned}
$$

In order to effectively distinguish the grey relational coefficient generated by two different time series, two comparative variables have been introduced into the formula (5), which are the subtraction variable $\left|y_{1}\left(t_{k}\right)-y_{2}\left(t_{k}\right)\right| / 2 \pi$ and the proportion variable $\left[1-\left(\min \left(\left|y_{1}\left(t_{k}\right)\right|,\left|y_{2}\left(t_{k}\right)\right|\right) \mid\right.\right.$ $\left.\left.\max \left(\left|y_{2}\left(t_{k}\right)\right|,\left|y_{2}\left(t_{k}\right)\right|\right)\right)\right]$. When the angle increments of the corresponding period of time $\left(\Delta t_{k}\right)$ in these two time series are equal or approximately equal, the subtraction variable and the proportion variable will be all approximately equal to 0 , and the relational coefficient generated by these two time series will be the largest, whose value is about 1; otherwise, it will be the smallest [5].

And if there are more than two time series, then we usually consider one of them as the reference series and the others as the comparative series, and their grey relational coefficients can be obtained by the formula (5).

Step 4 (calculating the grey relational degree). Assume there are only two time series on the interval $[p, q]$, their corresponding angle series are $A_{1}=\left\{\alpha_{1}\left(t_{2}\right), \alpha_{1}\left(t_{3}\right), \ldots, \alpha_{1}\left(t_{n-1}\right)\right\}$ and $A_{2}=\left\{\alpha_{2}\left(t_{2}\right), \alpha_{2}\left(t_{3}\right), \ldots, \alpha_{2}\left(t_{n-1}\right)\right\}$, respectively; the following formula will be defined to calculate the grey relational degree between $A_{1}$ and $A_{2}$ :

$$
r=\frac{1}{p-q} \sum_{k=3}^{n-1} \Delta t_{k} \cdot \xi\left(t_{k}\right) .
$$

Step 5 (ranking the relationship intensity). The relationship intensity between the comparative series and the reference series can be ranked according to their grey relational degrees, and the higher value indicates the higher intensity.

3.2. Discussion on Two Special Cases. The above process provides a novel thought to measure the grey relational degree between different time series. However, to some special cases in reality, we cannot calculate their grey relational degree using the above process directly, and we usually need to do some adjustments to these special cases or utilize some other methods to calculate their grey relational degree.

Case 1 (the "linear-type" time series). Assume there are two "linear-type" time series (Figure 3(a)), firstly, they can be expressed as the form of piecewise function with the same slope based on PLI, and then it can be transformed into the angle series with Step 1. However, since all angle values at every observation time point are all $\pi$, thus, if we calculate 
their grey relational degree according to Steps 2, 3, and 4, the result will be 1 , which is obviously unreasonable. Therefore, to this special case, we cannot calculate their grey relational degree using the above process; however, another model called slope relational degree developed by Dang can be considered.

Case 2 (the "time-dislocation-type" time series). Time series with similar properties usually have the same observation points including the number and the observation time; however, to some special cases, after they have been expressed as the form of the piecewise function, their observation time points are sometimes not corresponding, and the perpendicular projections of the line segments onto abscissa are also different (Figure 3(b)). Thus, some necessary adjustments usually need to be done firstly, and then their grey relational degrees can be measured with the above process.

Definition 2. Assume there are two time series, if their observation time points are not corresponding, then one calls them as the "time-dislocation-type" time series.

The measurement of grey relational degree of "timedislocation-type" time series is usually complex. Specially, the number and the observation time of time series should be unified firstly, and then the order of angles in different time series also needs to be adjusted according to their similarity, and the newly added angles are all be set to $\pi$, after this, the lengths of perpendicular projections of the corresponding time series onto abscissa will be consistent. Finally, their relational degrees can be measured using the model of GRAAP.

For instance, assume there are two time series, their corresponding angle series are $A_{1}=\left\{\alpha_{1}\left(t_{3}\right), \alpha_{1}\left(t_{4}\right)\right\}$ and $A_{2}=\left\{\alpha_{2}\left(t_{2}\right), \alpha_{2}\left(t_{5}\right)\right\}$, respectively (Figure 3(b)). Firstly, two new angles will be added into the corresponding time series, and these new angles are set as follows: $\alpha_{1}\left(t_{2}\right)=\alpha_{2}\left(t_{3}\right)=$ $\alpha_{2}\left(t_{4}\right)=\alpha_{1}\left(t_{5}\right)=\pi$. And then the position of angles needs to be adjusted according to their similarity, we can know that the similarity between $\alpha_{1}\left(t_{3}\right)$ and $\alpha_{2}\left(t_{2}\right)$ and $\alpha_{1}\left(t_{4}\right)$ and $\alpha_{2}\left(t_{5}\right)$ is high enough from Figure 3(b); therefore, these two time series will be changed as follows:

$$
\begin{aligned}
& A_{1}=\left\{\alpha_{1}\left(t_{2}\right), \alpha_{1}\left(t_{3}\right), \alpha_{1}\left(t_{4}\right), \alpha_{1}\left(t_{5}\right)\right\}, \\
& A_{2}=\left\{\alpha_{2}\left(t_{3}\right), \alpha_{2}\left(t_{2}\right), \alpha_{2}\left(t_{5}\right), \alpha_{2}\left(t_{4}\right)\right\} .
\end{aligned}
$$

After this, their relational degree can be calculated by applying the process of GRAAP. However, since the position of the observation time points of the time series has been adjusted, the result will obviously be affected to a certain extent; so the final relational degree will changed into $\rho r(0<$ $\rho<1)$.

\section{Property}

Theorem 3. The properties of the model of GRAAP are as follows:

(1) $|r| \leq 1$;

(2) symmetry, that is, $r\left(X_{1}, X_{2}\right)=r\left(X_{2}, X_{1}\right)$;

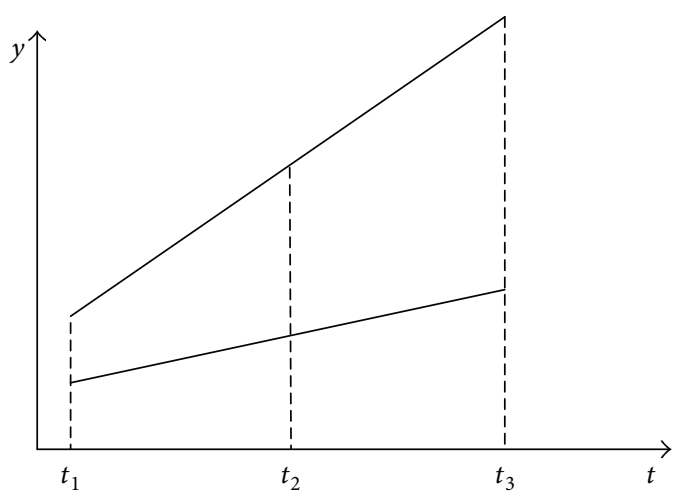

(a)

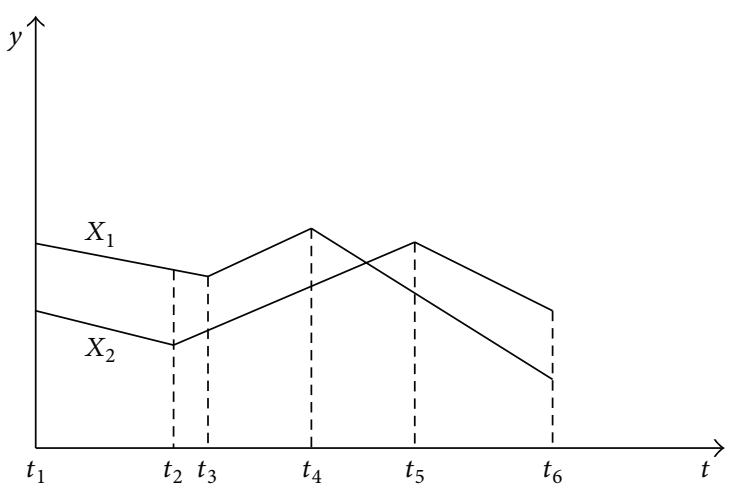

(b)

Figure 3: Diagrams of (a) "linear-type" time series and (b) "timedislocation-type" time series.

(3) uniqueness;

(4) comparability.

Proof. Consider the following.

Property (1). We can obtain $0<\alpha_{m}\left(t_{k}\right)<2 \pi$ from Definition 1, so $0<y_{m}\left(t_{k}\right)<2 \pi \Leftrightarrow-1 \leq \xi\left(t_{k}\right) \leq 1$, $k=3,4, \ldots, n$, and $[p, q]=\cup_{k=2}^{n} \Delta t_{k} \Leftrightarrow-1 \leq r=(1 /(q-$ p)) $\sum_{k=2}^{n} \Delta t_{k} \cdot \xi\left(t_{k}\right) \leq 1$.

Property (2). Because

$$
\begin{aligned}
r\left(X_{1}, X_{2}\right) & \\
= & \frac{1}{p-q} \sum_{k=3}^{n-1} \Delta t_{k} \cdot \xi\left(t_{k}\right) \\
= & \frac{1}{p-q} \sum_{k=3}^{n-1} \Delta t_{k} \\
& \times\left(1+\frac{\left|y_{1}\left(t_{k}\right)-y_{2}\left(t_{k}\right)\right|}{2 \pi}\right. \\
& \left.+\frac{1}{2}\left[1-\frac{\min \left(\left|y_{1}\left(t_{k}\right)\right|,\left|y_{2}\left(t_{k}\right)\right|\right)}{\max \left(\left|y_{1}\left(t_{k}\right)\right|,\left|y_{2}\left(t_{k}\right)\right|\right)}\right]\right)^{-1}
\end{aligned}
$$




$$
\begin{aligned}
& =\frac{1}{p-q} \sum_{k=3}^{n-1} \Delta t_{k} \\
& \quad \times\left(1+\frac{\left|y_{2}\left(t_{k}\right)-y_{1}\left(t_{k}\right)\right|}{2 \pi}\right. \\
& \left.\quad \quad+\frac{1}{2}\left[1-\frac{\min \left(\left|y_{2}\left(t_{k}\right)\right|,\left|y_{1}\left(t_{k}\right)\right|\right)}{\max \left(\left|y_{2}\left(t_{k}\right)\right|,\left|y_{1}\left(t_{k}\right)\right|\right)}\right]\right)^{-1} \\
& = \\
& \quad r\left(X_{2}, X_{1}\right),
\end{aligned}
$$

so Property (2) is correct.

Property (3). For one time series, its interval [p, $q], \Delta t_{k}$, and $\xi\left(t_{k}\right)$ are all usually unique according to the formula (5); so $r$ is also unique.

Property (4). This property is also correct due to Property (3).

Theorem 4. Assume there are two time series, that is, $X_{1}$ and $X_{2}$, and their grey relational degree is $r\left(X_{1}, X_{2}\right)$, which satisfies $\mid r\left(X_{1}, X_{2} \mid \leq 1\right.$ and $r\left(X_{1}, X_{2}\right)=1$, if and only if $X_{2}\left(t_{k}\right)=$ $X_{1}\left(t_{k}\right)+c(k=1,2, \ldots, n ; c$ is a constant $)$, then $X_{1}$ and $X_{2}$ are parallel, that is, the GRAAP model satisfies the normativity of GST.

Proof. Because $\mid r\left(X_{1}, X_{2} \mid \leq 1\right.$ and $r\left(X_{1}, X_{2}\right)=1 \Leftrightarrow \xi\left(t_{k}\right)=$ $1 \Leftrightarrow y_{1}\left(t_{k}\right)=y_{2}\left(t_{k}\right) \Leftrightarrow \alpha_{1}\left(t_{k}\right)-\alpha_{1}\left(t_{k-1}\right)=\alpha_{2}\left(t_{k}\right)-\alpha_{2}\left(t_{k-1}\right) \Leftrightarrow$ $\alpha_{1}\left(t_{k}\right)-\alpha_{1}\left(t_{1}\right)=\alpha_{2}\left(t_{k}\right)-\alpha_{2}\left(t_{1}\right) \Leftrightarrow \alpha_{2}\left(t_{k}\right)=\alpha_{1}\left(t_{k}\right)+\alpha_{1}\left(t_{1}\right)-$ $\alpha_{2}\left(t_{1}\right) \Leftrightarrow \alpha_{2}\left(t_{k}\right)=\alpha_{1}\left(t_{k}\right)+c \Leftrightarrow X_{2}\left(t_{k}\right)=X_{1}\left(t_{k}\right)+c \Leftrightarrow$, time series $X_{1}$ and $X_{2}$ are parallel.

And $c=\alpha_{1}\left(t_{1}\right)-\alpha_{2}\left(t_{1}\right)$.

That is, the model of GRAAP satisfies the characteristic of GRA's normativity.

So this property was proved.

\section{Case Study}

5.1. Case Analyses. As mentioned above, the novel model presented in this paper was mainly rooted in two traditional GRA models, that is, Grey T's Correlation Degree explored by Tang [12] and the Improved Grey T's Correlation Degree by Sun and Tang [5], and following the current naming habit and some researchers' advice, this novel model was named as grey relational analysis based on angle perspective (GRAAP). Currently, these two traditional models have been widely accepted by industry and academia, and they have also made some contributions to the real-world. Meanwhile, the model by Sun was rooted in the model by Tang and made some improvements, so it is generally believed that this model is more perfect. Similarly, the novel model in this paper was rooted in these two traditional models, and it can make more fully use of the inefficient information in grey system through the transformation to time series; so it can be considered as a more effective and reliable model. And in order to further prove its effectiveness, a comparative case study will be performed considering an example in [2], and four time
TABLE 1: Original time series from [2].

\begin{tabular}{lccccccc}
\hline Series & \multicolumn{7}{c}{ Time } \\
& $t_{1}$ & $t_{2}$ & $t_{3}$ & $t_{4}$ & $t_{5}$ & $t_{6}$ & $t_{7}$ \\
\hline $\mathbf{X}_{\mathbf{0}}$ & 1.0 & 2.0 & 2.5 & 2.5 & 3.0 & 5.0 & 6.0 \\
$\mathbf{X}_{1}$ & 1.0 & 1.8 & 2.3 & 2.4 & 2.8 & 4.8 & 5.8 \\
$\mathbf{X}_{2}$ & 1.0 & 1.8 & 2.3 & 2.0 & 3.0 & 4.1 & 5.7 \\
$\mathbf{X}_{3}$ & 1.0 & 2.19 & 2.5 & 2.1 & 3.0 & 4.25 & 5.8 \\
\hline
\end{tabular}

TABLE 2: The corresponding angle series of the original time series.

\begin{tabular}{lccccc}
\hline Series & \multicolumn{5}{c}{ Angle } \\
& $\alpha_{m}\left(t_{2}\right)$ & $\alpha_{m}\left(t_{3}\right)$ & $\alpha_{m}\left(t_{4}\right)$ & $\alpha_{m}\left(t_{5}\right)$ & $\alpha_{m}\left(t_{6}\right)$ \\
\hline $\mathbf{X}_{\mathbf{0}}$ & $1.3427 \pi$ & $1.6150 \pi$ & $0.3850 \pi$ & $0.7049 \pi$ & $1.1976 \pi$ \\
$\mathbf{X}_{1}$ & $1.3647 \pi$ & $1.5667 \pi$ & $0.4162 \pi$ & $0.6709 \pi$ & $1.1976 \pi$ \\
$\mathbf{X}_{2}$ & $1.3647 \pi$ & $1.7324 \pi$ & $0.2740 \pi$ & $0.7567 \pi$ & $0.7990 \pi$ \\
$\mathbf{X}_{3}$ & $1.3959 \pi$ & $1.7299 \pi$ & $0.2369 \pi$ & $0.7565 \pi$ & $0.8097 \pi$ \\
\hline
\end{tabular}

TABLE 3: The relational coefficients between $\mathbf{X}_{0}$ and other three time series.

\begin{tabular}{lcccc}
\hline Objects & $\xi\left(t_{3}\right)$ & $\xi\left(t_{4}\right)$ & $\xi\left(t_{5}\right)$ & $\xi\left(t_{6}\right)$ \\
\hline $\mathbf{X}_{\mathbf{0}}$ and $\mathbf{X}_{\mathbf{1}}$ & 0.8576 & 0.9328 & 0.8814 & 0.9530 \\
$\mathbf{X}_{\mathbf{0}}$ and $\mathbf{X}_{\mathbf{2}}$ & 0.8507 & 0.8386 & 0.8000 & 0.5944 \\
$\mathbf{X}_{\mathbf{0}}$ and $\mathbf{X}_{\mathbf{3}}$ & 0.8919 & 0.8200 & 0.7740 & 0.6003 \\
\hline
\end{tabular}

series have been analyzed in this paper; the original time series were as in Table 1.

The corresponding angle series of the original time series were as in Table 2 with Step 1.

The relational coefficient $\xi\left(t_{k}\right)$ can be obtained with the Steps 2, 3, and 4. Specially, the paper will consider $\mathbf{X}_{\mathbf{0}}$ as the reference series, and $\mathbf{X}_{1}, \mathbf{X}_{2}$, and $\mathbf{X}_{3}$ were the comparative series; the relational coefficients between $\mathbf{X}_{\mathbf{0}}$ and other three time series were as shown in Table 3.

Finally, the grey relational degrees between $\mathbf{X}_{0}$ and other three time series could be obtained with Step 4 . Consider the following:

$$
\begin{aligned}
& r_{01}=\frac{1}{p-q} \sum_{k=3}^{n-1} \Delta t_{k} \cdot \xi\left(t_{k}\right)=0.9062, \\
& r_{02}=\frac{1}{p-q} \sum_{k=3}^{n-1} \Delta t_{k} \cdot \xi\left(t_{k}\right)=0.7709 \\
& r_{03}=\frac{1}{p-q} \sum_{k=3}^{n-1} \Delta t_{k} \cdot \xi\left(t_{k}\right)=0.7715
\end{aligned}
$$

5.2. Discussion. The relationship between $\mathbf{X}_{0}$ and other three time series based on the above grey relational degrees was as follows:

$$
r_{01}>r_{03}>r_{02} .
$$

The results obtained by GRAAP indicated that the grey relational degree between $\mathbf{X}_{\mathbf{0}}$ and $\mathbf{X}_{\mathbf{1}}$ was the highest, and 
TABLE 4: Result comparisons of three different methods.

\begin{tabular}{lcccc}
\hline Method & & $r$ & & Source \\
\hline $\begin{array}{l}\text { The grey relational model based on the angle } \\
\text { perspective }\end{array}$ & 0.9062 & 0.7715 & 0.7709 & Presented in this paper \\
$\begin{array}{l}\text { Improvement model on grey T's relational degree } \\
\begin{array}{l}\text { The concept and the computation method of T's } \\
\text { relational degree }\end{array}\end{array}$ & 0.8712 & 0.7147 & 0.7063 & Presented in [2] \\
\hline
\end{tabular}

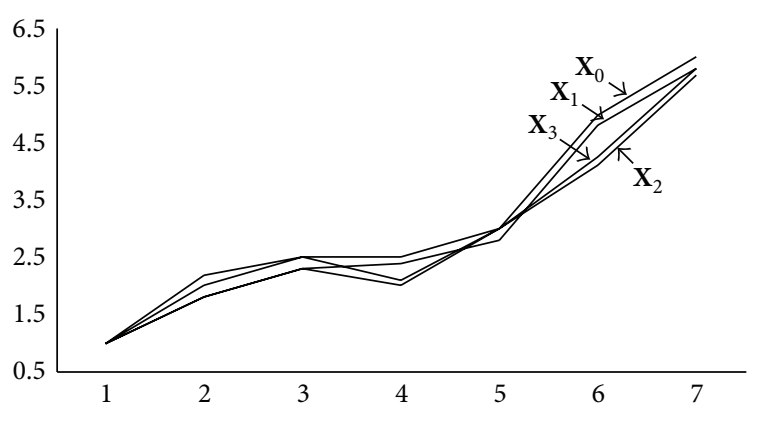

FIGURE 4: Geometry curves of four time series.

the degree between $\mathbf{X}_{0}$ and $\mathbf{X}_{2}$ was the smallest, whose value was approximately equal to that between $\mathbf{X}_{\mathbf{0}}$ and $\mathbf{X}_{3}$.

The following table showed the results obtained by three methods including GRAAP in this paper, improvement model on Grey T's Relational Degree in [2], and the Concept and the computation method of T's Relational Degree in [8].

The comparative results in the above table indicated that the difference between $r_{01}$ and $r_{02}$ was so high that their sort order obtained by three models was consistent (Table 4), that is, $r_{01}>r_{02}$; by contrast, the sort orders of $r_{02}$ and $r_{03}$ were different by these three models due to their approximately equal values.

The reason for this results was because the model in this paper was based on the thought of [2], which had addressed some shortcomings of the model in [8], that is to say, the models in this paper and [2] were obviously superior to the model in [8], and the results obtained by the model of GRAAP and the model in [2] were also more accurate and reasonable than that in [8], therefore, the sort order of $r_{01}$, $r_{02}$, and $r_{03}$ should be $r_{01}>r_{03}>r_{02}$.

And compared with the similarities of the geometry curves generated by these four time series shown in Figure 4, the similarity of geometry curves between $\mathbf{X}_{0}$ and $\mathbf{X}_{1}$ was highest, and the similarities between $\mathbf{X}_{0}$ and other two time series, $\mathbf{X}_{2}$ and $\mathbf{X}_{3}$, were approximate and relatively small, these results were consistent with that in Table 4 .

We can conclude that GRAAP presented in this paper was reasonable and effective from the above two comparable results in Table 4 and Figure 4, and this model, like other GRA models, can also be applied to make prediction, classification, and so forth.

\section{Conflict of Interests}

The authors declare that there is no conflict of interests regarding the publication of this paper.

\section{Acknowledgment}

This work was supported by the National Natural Science Foundation of China (no. 71272049) and Specialized Research Fund for the Doctoral Program of Higher Education of China (no. 20126102110052).

\section{References}

[1] R. Roostaee, M. Izadikhah, and F. H. Lotfi, "An interactive procedure to solve multi-objective decision-making problem: an improvement to STEM method," Journal of Applied Mathematics, vol. 2012, Article ID 324712, 18 pages, 2012.

[2] A. M. Yusof and S. Ismail, "Multiple regression analysis as a tool to property investment decision making," in Proceedings of the 17th IBIMA Conference on Creating Global Competitive Economies: A 360-Degree Approach, K. S. Soliman, Ed., vol. 1-4, pp. 700-708, Milan, Italy.

[3] J.S. Ma, "Grey target decision method for a variable target centre based on the decision maker's preferences," Journal of Applied Mathematics, vol. 2014, Article ID 572529, 6 pages, 2014.

[4] S. F. Liu, H. Cai, Y. J. Yang, and Y. Cao, "Advance in grey incidence analysis modelling," Systems Engineering-Theory and Practice, vol. 33, no. 8, pp. 2041-2046, 2013.

[5] Y.-G. Sun and Y.-G. Dang, "Improvement on grey T's correlation degree," Journal of System Science and Information, vol. 4, no. 4, pp. 135-139, 2008.

[6] Z.-X. Wang, "Nonlinear grey prediction model with convolution integral NGMC $(1, n)$ and its application to the forecasting of China's industrial $\mathrm{SO}_{2}$ emissions," Journal of Applied Mathematics, vol. 2014, Article ID 580161, 10 pages, 2014.

[7] S. F. Liu and Y. Lin, Grey Information: Theory and Practical Applications, Springer, 2006.

[8] B. Su, L. Liu, and T. F. Yang, "Research of artifical neural network forecasting model based on grey relational analysis," Journal of Systems Science \& Information, vol. 28, no. 9, pp. 98-104, 2008.

[9] J. L. Deng, "Introduction to grey system theory," The Journal of Grey System, vol. 1, no. 1, pp. 1-24, 1989.

[10] X. M. Li, Y. G. Dang, S. Ding, and J. Zhang, "Grey accumulation generation relational analysis model for nonequidistance unequal-length sequences and its application," Mathematical Problems in Engineering, vol. 2014, Article ID 764857, 8 pages, 2014. 
[11] J. L. Deng, "Spread of grey relational space," Journal of Grey Systems, vol. 7, no. 3, pp. 96-100, 1995.

[12] W. X. Tang, "The concept and the computation method of T's correlation degree," Journal of Applied Statistics and Management, vol. 14, no. 1, pp. 34-37, 1995.

[13] K. Zhang, W. Ye, and L. Zhao, "The absolute degree of grey incidence for grey sequence base on standard grey interval number operation," Kybernetes, vol. 41, no. 7-8, pp. 934-944, 2012.

[14] Q. Y. Wang, “The grey relational analysis of B-modes," Journal of Huazhong University of Science and Technology, vol. 17, no. 6, pp. 77-82, 1987.

[15] Q. Y. Wang and X. H. Zhao, "The relational analysis of C-model," Journal of Huazhong University of Science and Technology, vol. 27, no. 3, pp. 75-77, 1999.

[16] Z. G. Mei, "The concept and computation method of grey absolute correlation degree," Systems Engineering, vol. 10, no. 5, pp. 43-44, 1992.

[17] Y. G. Dang, S. F. Liu, B. Liu, and C. M. Mi, "Improvement on degree of grey slope incidence," Engineering Science, vol. 6, no. 3, pp. 41-44, 2004.

[18] S. F. Liu, "Generalized degree of grey incidence," in Information and System, Z. Shengkai, Ed., pp. 113-116, Dalian DMU Publishing House, 1992.

[19] R. Guimerà, A. Arenas, A. Díaz-Guilera, and F. Giralt, "Dynamical properties of model communication networks," Physical Review E, vol. 66, no. 2, Article ID 026704, 2002.

[20] J. Cui, "A novel grey relational degree and its application," Statistics and Decision, no. 20, pp. 14-16, 2008.

[21] J. Cui, Y. G. Dang, and S. F. Liu, "Novel properties of some grey relational analysis models," Systems Engineering, vol. 27, no. 4, pp. 65-70, 2009.

[22] N. M. Xie and S. F. Liu, "The parallel and uniform properties of several relational models," Systems Engineering, vol. 25, no. 8, pp. 98-103, 2007. 


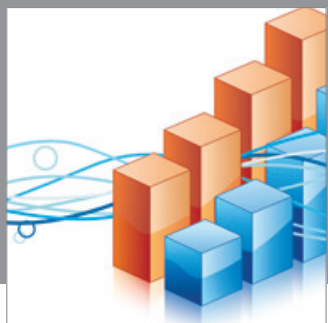

Advances in

Operations Research

mansans

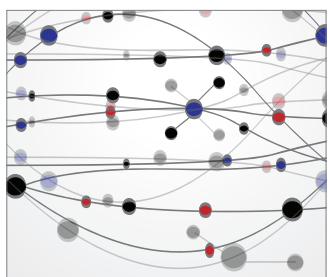

The Scientific World Journal
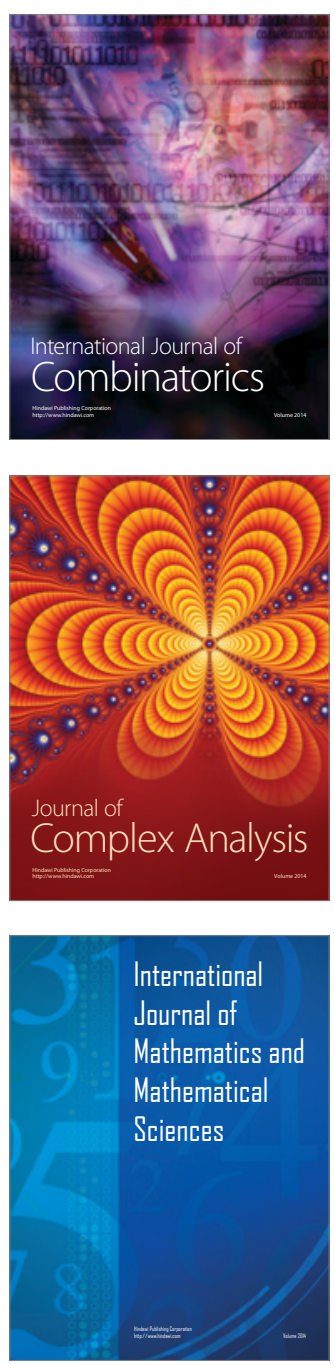
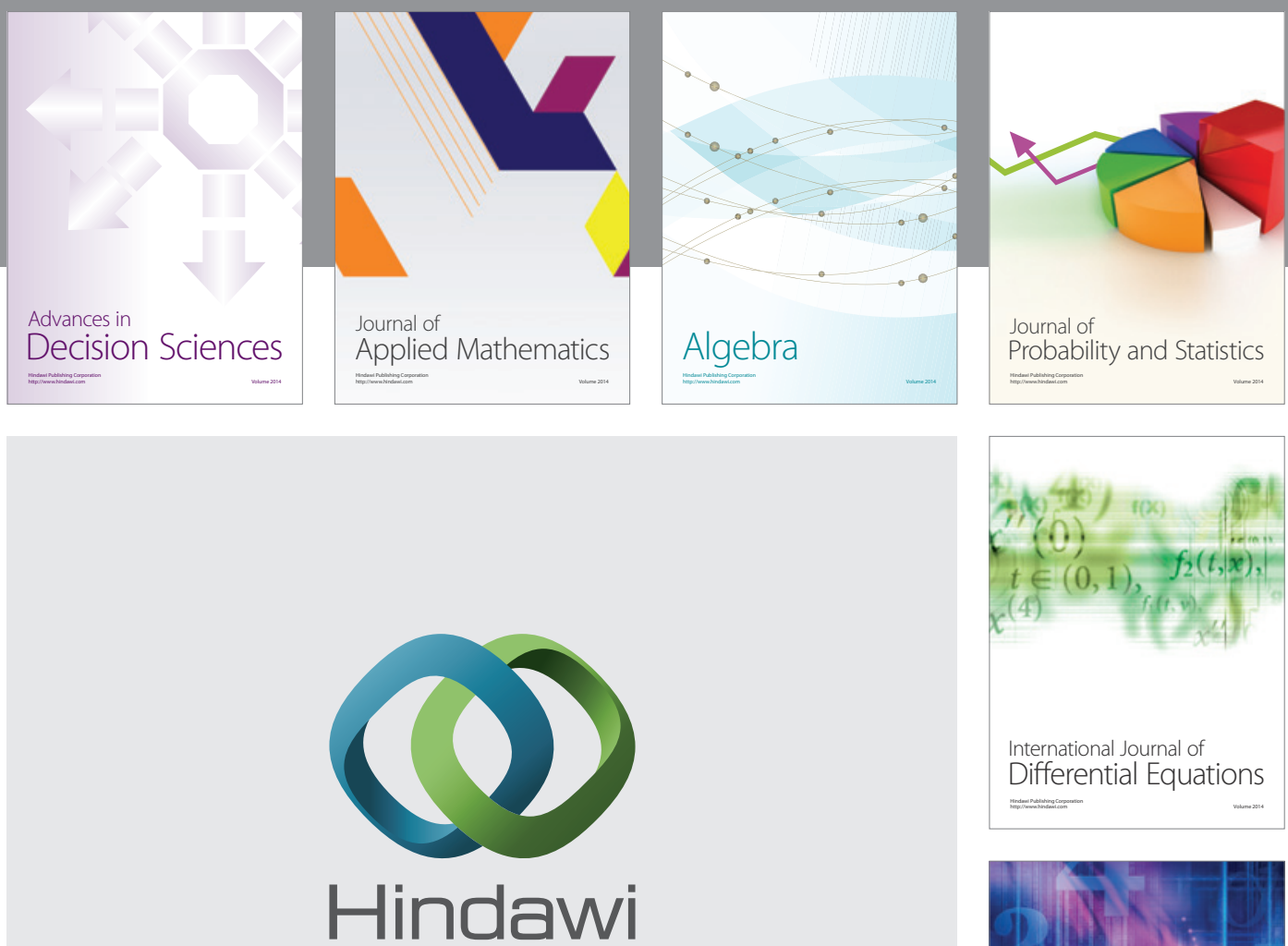

Submit your manuscripts at http://www.hindawi.com
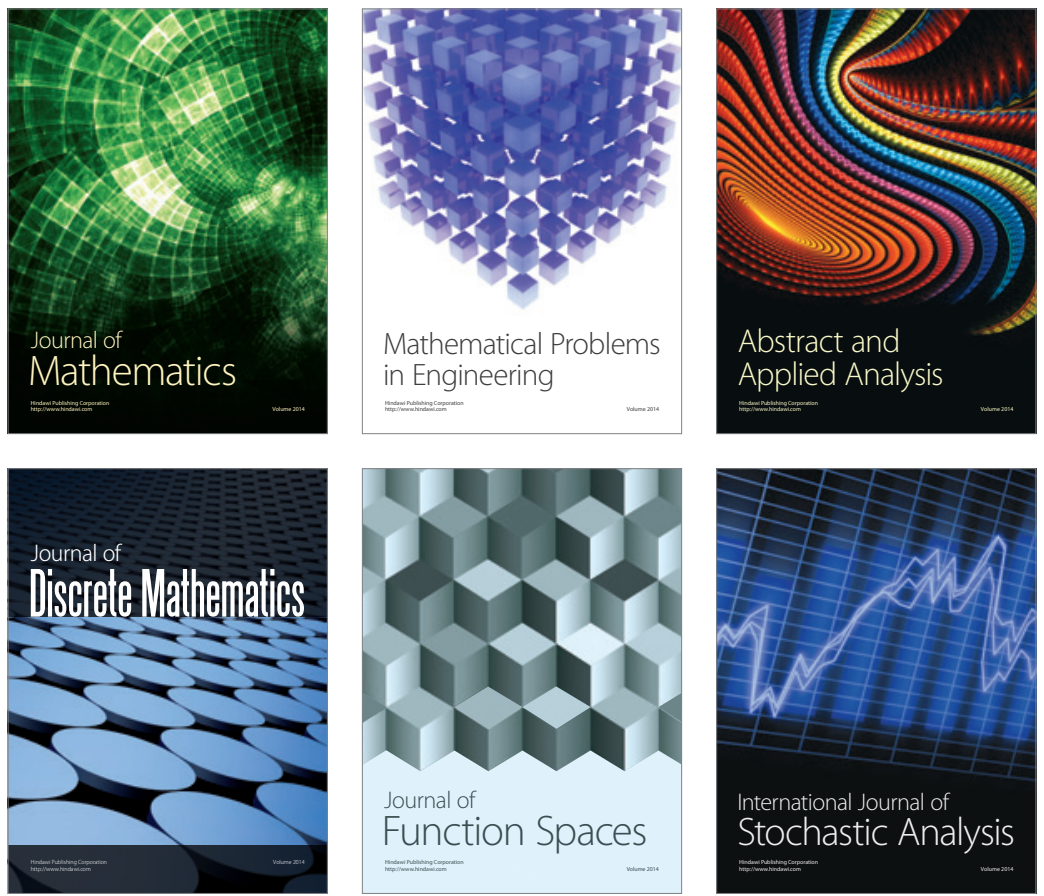

Journal of

Function Spaces

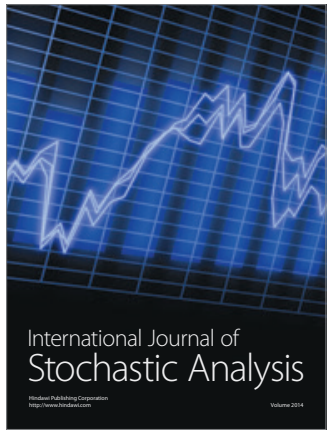

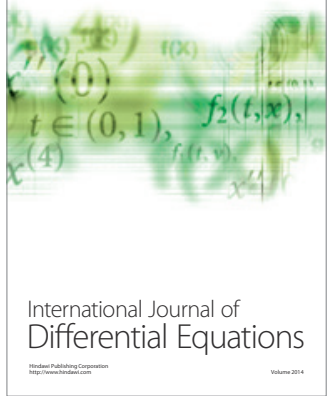
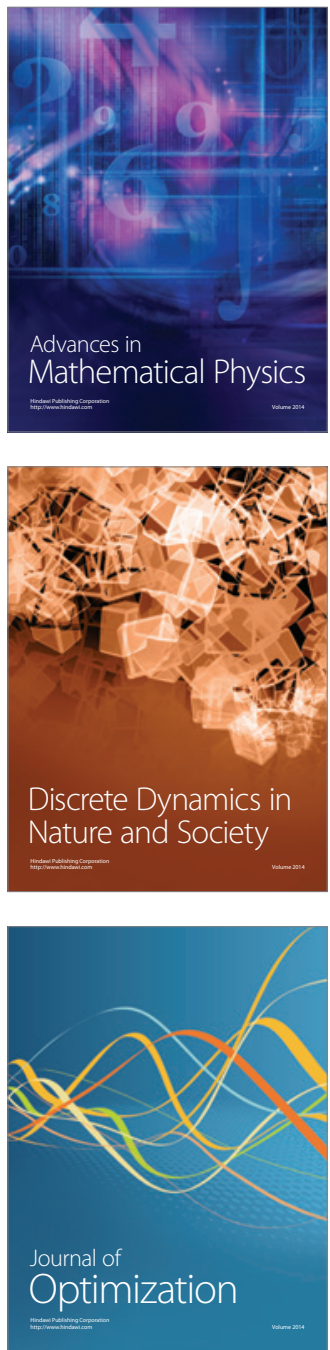